\title{
Resiliencia-Autoestima-Bienestar psicológico y Capacidad intelectual de estudiantes de cuarto medio de buen rendimiento de liceos vulnerables*
}

\author{
Resilience-Self Esteem-Psychological Well-Being and Intelectual Capacity of senior high \\ school students who have high perfomances in vulnerable schools \\ Resiliência-Autoestima-Bem-estar psicológico e Capacidade intelectual de estudantes \\ de quarto ano do ensino médio de liceus de vulnerabilidade e com bom desempenho \\ acadêmico
}

\section{Mario Morales N., ${ }^{a}$ Adolfo González G.}

aEscuela de Psicología, Facultad de Humanidades, Universidad de Santiago. Telf.: (562) 27184354. Correo electrónico: mario.morales@usach.cl

\section{RESUMEN}

Este artículo es un estudio realizado con 175 estudiantes de cuarto año medio de liceos prioritarios, con un alto índice de vulnerabilidad, perteneciente a la VI Región de Chile. Se evaluó su capacidad intelectual, resiliencia, bienestar psicológico y la autoestima. Se correlacionaron las distintas variables con el rendimiento escolar y los resultados obtenidos indican la existencia de correlación entre el bienestar psicológico y la resiliencia; la autoestima y la resiliencia; el bienestar psicológico y la autoestima, pero estas no se correlacionan con el rendimiento escolar. Posteriormente, se clasificaron los estudiantes en tres grupos: alumnos de alto rendimiento, de rendimiento promedio y un tercer grupo con bajo rendimiento. Se encontró diferencias significativas entre los grupos en la capacidad intelectual, pero no en las variables relacionadas con la autoestima, bienestar psicológico y resiliencia. $\mathrm{Si}$ se evidencia diferencias en algunos subfactores de las escalas administradas.

Palabras clave: liceos vulnerables, alto rendimiento, resiliencia, autoestima capacidad intelectual, bienestar psicológico.

\begin{abstract}
This article is a study of 175 seniors in high school from priority schools, with a high vulnerability index belonging to the VI Region of Chile. Their intellectual capacity, resilience, psychological well-being and self-esteem were assessed. There was a correlation between the different variables and school performance, and the results indicate the existence of a correlation between psychological well-being and resilience, self-esteem and resilience; psychological well-being and self-esteem, but there is no evidence of correlations with school performance. Subsequently, students were classified into three groups: high-performance, average, and a third group with low performance. There were found significant differences between the groups in their intellectual capacity, but not in the variables related to self-esteem, psychological well-being and resilience. There is evidence of differences among some subfactor of the administered scales.
\end{abstract}

Key words: vulnerable high schools, high academic performance, resilience, self esteem, intellectual ability, psychological well-being.

\section{RESUMO}

Estudo realizado com 175 estudantes do quarto ano do Ensino Médio de liceus prioritários, pertencentes a VI Região do Chile e com alto índice de vulnerabilidade. Avaliou-se a capacidade intelectual, resiliência, bem-estar psicológico e 
autoestima. Tais variáveis foram correlacionadas com o rendimento escolar e os resultados obtidos indicam a existência de uma correlação entre bem-estar psicológico e resiliência; entre autoestima e resiliência; entre bem-estar psicológico e autoestima, mas estas não se correlacionam com o rendimento escolar. Posteriormente, os estudantes foram classificados em três grupos: alunos com alto rendimento, com médio rendimento e com baixo rendimento. Entre os grupos, diferenças significativas foram encontradas em relação à capacidade intelectual, mas não nas variáveis relacionadas com a autoestima, bem-estar psicológico e resiliência. Evidenciam-se diferenças em alguns subfatores das escalas aplicadas.

Palavras chave: liceus vulneráveis, alto rendimento, resiliência, autoestima, capacidade intelectual, bem-estar psicológico.

\section{INTRODUCCIÓN}

El objetivo del presente trabajo es analizar las variables que determinan el alto rendimiento que presentan un grupo de estudiantes de cuarto año de enseñanza media provenientes de liceos prioritarios. El concepto de prioritarios es un término utilizado por el Ministerio de Educación de Chile y se refiere a aquellas instituciones escolares que debían ser intervenidas y apoyadas por Universidades debido a que presentaban diversos tipos de insuficiencias, tales como: prácticas de gestión directivas y de enseñanza pedagógica altamente deficientes, debilidad en equipos directivos, técnicos y docentes, conflictividad constante entre estudiantes y docentes, como entre docente y directivos, bajos rendimientos en pruebas nacionales. Entre las características más comunes encontradas en estos establecimientos es la falta de expectativa de los profesores respecto a la capacidad de aprendizaje de sus alumnos, que sumada a las difíciles condiciones de desempeño profesional no permitían visualizar posibilidades de cambio institucional (MINEDUC, 2006).

Por otra parte, la precariedad de las condiciones de vida en que están insertos estos estudiantes, operan en ellos altos niveles de riesgo en su entorno socio cultural, donde se evidencia circunstancias familiares, sociales, legales y culturales que obstaculizan el desarrollo personal. El concepto de vulnerabilidad se aplica a aquellos sectores o grupos que por su condición de edad, sexo, estado civil, origen étnico y situación socio económica se encuentran en condición de riesgo, lo que les impide incorporarse al desarrollo del país y acceder a mejores condiciones de bienestar.

El estudio permitió analizar diversos factores que pudiesen incidir en el alto rendimiento escolar de estudiantes de cuarto año medio pertenecientes a estos sectores de vulnerabilidad, considerando determinadas características cognitivas, afectivas y emocionales.

Las preguntas centrales de este estudio son: ¿qué factores favorecen a estudiantes de enseñanza media que a pesar de encontrarse insertos en liceos con alto índice de vulnerabilidad logran alcanzar altos rendimientos escolares? ¿Qué diferencias existe entre aquellos estudiantes de altos rendimientos con el grupo general en los factores intelectuales, resiliencia, autoestima y bienestar psicológico?

Las hipótesis que sustentan este estudio son: Existen diferencias significativas entre los grupos de estudiantes de alto rendimiento con el grupo general en variables relacionadas con la capacidad intelectual, resiliencia, autoestima y bienestar psicológico. La segunda hipótesis tiene que ver con la existencia de correlaciones positivas entre el rendimiento escolar y las variables intelectuales, resiliencia, autoestima y bienestar psicológico.

Entre las características cognitivas se analizó el rendimiento intelectual con el propósito de evaluar la capacidad intelectual, lo que permitió proporcionar información de la magnitud individual de algunas funciones cognoscitivas. En general, los estudios han 
demostrado una correlación positiva entre los factores intelectuales y el rendimiento escolar, sin embargo, esto no ha sido suficiente para explicar el éxito o el fracaso escolar. El análisis de esta relación nos señala que la aptitud verbal posee mayor capacidad predictiva del rendimiento escolar, los factores lingüísticos y psicolingüísticos presentarían una relación estrecha con los resultados escolares (Sternberg y Castejón, 1999; Canales, 2008; Padierna, Oseguera y Gudino, 2009).

El autoestima de los estudiantes fue otro factor estudiado, conceptualizada como la evaluación que la persona realiza y que mantiene en relación a sí misma (Coopersmith, 1987, cit. en Ramos, 2004), la percepción de la capacidad personal y de tener valor como persona (Polleti y Dobbs, 2005), valor del yo ante la percepción propia y de otras personas la cual se construye a lo largo de la vida (Naranjo, 2007). También se refieren a ella en términos de resultados, conceptualizada como el producto de un proceso dinamizador, alimentado por pensamientos y sentimientos de valoración personal (Romero, 2005) o el componente afectivo-evaluativo del auto concepto (García, 1983).

Otros de los aspectos considerados en este estudio fue la resiliencia. El supuesto que se sostuvo fue que aquellos estudiantes de enseñanza media de sectores vulnerables que presentarían mejor rendimiento escolar serían aquellos que han podido enfrentar con éxito una serie de obstáculos contextuales.

En la psicología positiva la resiliencia es entendida como una adaptación positiva frente a los riesgos o adversidades (Masten, 2001). Luhtans (2002) se refiere a la capacidad psicológica para recuperarse de los conflictos, adversidad, incertidumbre y fracaso. Es la capacidad de recuperarse, sobreponerse y adaptarse con éxito frente a la adversidad, esto implica desarrollar competencia social, académica y vocacional, pese a estar expuesto a un estrés grave o simplemente a las tensiones inherentes al mundo actual (Henderson y Milstein, 2003; Csikszentnihalyi, 1999; Grotberg, 1995; Rutter, 1985; Amar, J., Kotlianrenko, y Abello, 2003). Mruk (1999) establece una relación entre la autoestima y el éxito, sin embargo, enfatiza que este factor es extremadamente relevante para la superación de tales retos vitales.

El bienestar psicológico es entendido como el grado en que un individuo juzga su vida como un todo en términos favorables y satisfactorios. De acuerdo a Veenhoven (1994); el bienestar se caracteriza por tres elementos: su carácter subjetivo, que descansa sobre la propia experiencia de la persona, su dimensión global, porque incluye la valoración de todas las áreas de su vida y la apreciación positiva, que va más allá de la ausencia de factores negativos. Por su parte, Diener (1994), lo asocia a estados de humor positivo, alta autoestima y baja sintomatología depresiva. Ryff (1989) desarrolla un marco multidimensional del Bienestar Psicológico, estableciendo seis dimensiones, entre las cuales se menciona: autoaceptación, dominio del ambiente, sentimiento de realización en la vida, compromiso vital, autonomía y vínculos. Castro (2010) considera que el bienestar psicológico posee tres componentes básicos: afecto positivo, afecto negativo y el componente cognitivo. Este último es el resultado de la integración cognitiva que las personas realizan al evaluar de manera permanente como les está yendo o como les fue durante el transcurso de su vida. Por lo cual, el bienestar sería un juicio cognitivo.

Estos factores anteriormente descritos -rendimiento intelectual, autoestima, bienestar psicológico y resiliencia- se analizaron en virtud de la relación que pudiesen tener con el rendimiento escolar y se comparó si estos factores se diferenciaban entre los grupos con distintos promedios escolares. 


\section{METODOLOGÍA}

Se trata de un estudio cuantitativo correlacional con un diseño no experimental transeccional, en el cual se analizaron diversas variables en un momento determinado. El total de estudiantes evaluados fueron 175, todos cursando el cuarto año medio, pertenecientes a dos liceos prioritarios de la Sexta Región del País. La edad promedio de los jóvenes era de 18,58 años y una desviación estándar de 0.66. El 65\% eran varones y un $35 \%$ mujeres.

Los instrumentos aplicados fueron los siguientes:

-Raven's Standard Progressive Matrices (PMS). Evalúa la capacidad del sujeto para comprender y aplicar el (los) principio(s) que subyace(n) en la resolución de problemas. Evalúa el factor " $\mathrm{g}$ " de la inteligencia mediante pruebas que no son verbales. La decisión de utilizar una prueba no verbal, fue por la limitación que poseen estos jóvenes en el desarrollo de las áreas del lenguaje y razonamiento lógico matemático, factores fundamentales que determinan la inteligencia cristalizada. En este test, el sujeto debe deducir relaciones entre algunas figuras geométricas y luego encontrar el correlato adecuado. No es un test de CI, sino de habilidad cognitiva para abstraer elementos visuales que configuran un gráfico de forma más o menos compleja. El nivel de confiabilidad del instrumento es de 0.78 (Alfa de Cronbach).

-Inventario de autoestima de Coopersmith. Es una prueba individual y colectiva y está dividido en los siguientes sub test: Sub test Sí Mismo, en el cual los puntajes altos indican valoración de sí mismo y altos niveles de aspiración, estabilidad, confianza, adecuadas habilidades sociales y atributos personales. Sub test Social Pares, una puntuación alta indica que el sujeto posee mayores competencias y habilidades en las relaciones con amigos y colaboradores, así como con extraños. Sub test Hogar Padres, un nivel alto revela buenas cualidades y habilidades en las relaciones íntimas con la familia, el sujeto se siente respetado, tiene independencia y una concepción moral propia. Sub test escolar, los niveles altos indican que el individuo afronta adecuadamente las principales tareas académicas y son personas que poseen buena capacidad para aprender, trabajan la satisfacción tanto a nivel individual como grupal y un Sub test L (Mentira), donde los altos puntajes indicaría falta de consistencia en los resultados por lo que el inventario quedaría invalidado. El nivel de confiabilidad es de 0,73 (Alfa de Cronbach).

-Test de Resiliencia. La escala de resiliencia SV-RES para jóvenes y adultos (Saavedra y Villalta, 2008) se basa teóricamente en los modelos sobre el constructo de resiliencia de los autores Grotberg (1996) y Saavedra (2003). Fue validada estadísticamente el año 2007, probándose en población urbana chilena entre 15 y 65 años. Es un instrumento que puede ser aplicado individual y colectivamente e incluso puede ser auto administrado. Cuenta con 60 ítems con cinco alternativas en escala likert, que van desde "muy de acuerdo" a "muy en desacuerdo". Permite obtener un puntaje global de resiliencia y un puntaje en las siguientes áreas: identidad, autonomía, satisfacción, pragmatismo, vínculos, redes, modelos, metas, afectividad, autoeficacia, aprendizaje y generatividad. El nivel de confiabilidad de la escala es de 0,74 (Alfa de Cronbach).

-Escala de Bienestar Psicológico (Bieps) desarrollada por Casullo (2000), consta de 13 reactivos, cuyas puntuaciones van de 1 (en desacuerdo) hasta 3 (de acuerdo). Las dimensiones que evalúa son: control, vínculos, proyectos de vida, aceptación. El instrumento posee un nivel de confiabilidad y validez adecuado. Se ha utilizado en otras 
investigaciones relacionadas en estudios con adolescentes. El nivel de confiabilidad es de 0,69 (Alfa de Cronbach).

-El rendimiento escolar fue el promedio obtenido entre el segundo y cuarto año de enseñanza media.

Se obtuvieron los promedios de notas de todos los participantes y se les administró una batería de pruebas, anteriormente descritas. Los alumnos se agruparon en tres grupos a partir de los promedios de notas: 1) Grupo 1, aquellos estudiantes de alto rendimiento cuyos promedios se encontraban a una desviación estándar sobre el promedio del grupo general; 2) Grupo 2, estudiantes que se ubicaban en el promedio del curso; y 3) Grupo 3 constituido por aquellos que presentaban una desviación estándar bajo el promedio del grupo total. Posteriormente, se compararon los promedios obtenidos por los tres grupos para determinar la existencia de diferencias significativas entre las variables estudiadas.

\section{RESULTADOS}

\subsection{DESCRIPCIÓN DE LAS VARIABLES EN EL GRUPO TOTAL}

El cuadro 1 provee las medias y desviaciones estándar de cada una de las variables analizadas. Se consideraron los promedios de todo el grupo estudiado ( $\mathrm{N}=175)$. El promedio del grupo estudiado es de 5,49; este se calculó considerando el rendimiento de todas las asignaturas de los cursos tercero y cuarto medio.

Cuadro 1. Medias y Desviaciones Estándar de las variables estudiadas

\begin{tabular}{|c|c|c|}
\hline Factor & Media & D.E. \\
\hline Rendimiento escolar & 5.49 & 0,45 \\
\hline Autoestima* & 51,14 & 9,9 \\
\hline Rendimiento Intelectual* & 42.14 & 6,1 \\
\hline $\begin{array}{c}\text { Bienestar Psicológico. } \\
\text { BIEPS** }\end{array}$ & 34,79 & 3,7 \\
\hline Resiliencia* & 53.6 & 25,1 \\
\hline Grupo total & 175 & \\
\hline
\end{tabular}

Con relación al test de autoestima, se obtiene un T de 51,14, ubicándose el puntaje dentro de la norma estadística. Los factores que evalúa el test (Cuadro 2) poseen todos un valor cercano al promedio.

Cuadro 2. Escala de Autoestima

\begin{tabular}{|c|c|c|}
\hline Factor & Media & D.E. \\
\hline General & 50,7 & 8.9 \\
\hline Social* $^{*}$ & 54,4 & 11,7 \\
\hline
\end{tabular}




\begin{tabular}{|c|c|c|}
\hline Hogar & 46,4 & 8,5 \\
\hline Escolar & 54,7 & 10,5 \\
\hline Escala de Mentira & 46,6 & 22,1 \\
\hline Grupo total & 175 & \\
\hline
\end{tabular}

En el subtest general, el puntaje obtenido se encuentra dentro de los límites normales. Los resultados dan cuenta de la valoración de sí mismo, niveles de aspiración, estabilidad frente los desafíos, confianza, habilidades sociales y atributos personales. Respecto al subtest de autoestima social las puntuaciones se encuentran dentro de la normalidad estadística, las habilidades se relaciona con el desenvolvimiento dentro de la comunidad, enfrentamientos adecuados a los problemas sociales, relación con los amigos y sentido de pertenencia. Los resultados en el subtest de hogar padres, está constituido por indicadores relacionadas con relaciones íntimas con la familia, respeto y consideración, independencia, aspiraciones familiares, valores familiares, pese a que es el resultado más bajo los puntajes obtenidos se encuentran dentro de la normalidad estadística. Finalmente, en el subtest relacionado con autoestima escolar también se encuentra dentro de los límites normales, existe capacidad para aprender, afrontar las tareas académicas, evaluación de los propios logros, planteamientos de metas superiores, satisfacción de trabajar en forma grupal e individual. Con respecto a la escala de mentira, el resultado indica que las respuestas son confiables y válidas.

Respecto al rendimiento cognitivo, evaluado por el test de Raven (matrices progresivas), se puede señalar que el promedio obtenido por el grupo corresponde a un T 42, encontrándose a 0,8 desviaciones estándar bajo el promedio del grupo de referencia, pero estadísticamente normal.

En cuanto al bienestar psicológico, la puntuación total corresponde a un percentil de 34,7 evidenciándose un rendimiento bajo, cerca de un 66\% del grupo estandarizado se encontraría sobre el rendimiento de este grupo. El análisis por área se puede observar en el Cuadro 3.

Cuadro 3. Bienestar Psicológico

\begin{tabular}{|c|c|c|}
\hline Factor & Media & D.E. \\
\hline Control & 10,7 & 1,2 \\
\hline Vínculo & 8,1 & 0,9 \\
\hline Proyecto & 8,3 & 1,0 \\
\hline Autonomía & 8,0 & 1,1 \\
\hline
\end{tabular}

Los rendimientos se encuentran dentro de los límites normales en todas las áreas evaluadas. La dimensión control de la escala tiene relación con auto competencia, posibilidades de crear o manipular contextos para adecuarlos a sus propias necesidades. Son capaces de manejar los asuntos de la vida diaria, conciencia de las oportunidades y capaces de modificar el ambiente. Los reactivos de la escala son: Si algo sale mal ser capaz de aceptarlo, el poder aceptar las equivocaciones y tratar de mejorar y encarar sin mayores problemas las obligaciones diarias. 
Con relación al vínculo, podemos señalar que es una puntuación alta y se relaciona con la capacidad de aceptar los múltiples aspectos de sí mismo, incluyendo aquellos positivos como negativos. Son sujetos que se sienten bien acerca del pasado, satisfechos consigo mismo. Los reactivos tienen que ver con: Percepción de caerle bien a la gente, contar con personas cuando uno las necesita, llevarse bien con los demás.

La dimensión proyecto también aparece alta. Esto se relaciona con metas y proyectos futuros. Los reactivos son: Saber lo que quieren hacer en la vida, sobre el que hacer en el futuro, lograr las metas propuestas, capaz de pensar en un proyecto para su vida personal

Finalmente, en la dimensión autonomía se pudo apreciar que los jóvenes son capaces de tomar decisiones de modo independiente, confiar en su propio juicio y además aparece cierta estabilidad emocional. Los indicadores se relacionan con: expresar lo que se piensa sin problemas, hacer lo que se quiere sin ser influenciable y tomar decisiones sin mayores dudas.

Los resultados en test de resiliencia, aparece con un $\mathrm{T}(53,6)$, que se encuentra dentro de los límites normales. Respecto a los subtest de la escala, se puede apreciar en el cuadro 4, que el factor modelo es aquel que presenta una puntuación mayor. El subtest de la escala tiene relación con juicios que se refieren a la convicción del papel de las redes sociales cercanas para apoyar la superación de situaciones problemáticas nuevas.

Mientras que el subtest de satisfacción aparece como el más bajo (aunque dentro de la norma estadística). Se refiere a juicios que develan la forma particular en como el sujeto interpreta una situación problemática, percepción de logro, autovaloración, adaptación efectiva a las condiciones ambientales y percepción de desarrollo.

Cuadro 4. Escala de Resiliencia

\begin{tabular}{|c|c|c|}
\hline Factor & Media & D.E. \\
\hline Identidad & 60,2 & 25,9 \\
\hline Autonomía & 60,9 & 26,9 \\
\hline Satisfacción & 51,4 & 26,4 \\
\hline Pragmatismo & 56,9 & 26,7 \\
\hline Vínculos & 53,8 & 27,9 \\
\hline Redes & 55,6 & 28,0 \\
\hline Modelos & 62,6 & 27,5 \\
\hline Metas & Media & D.E. \\
\hline Afectividad & 57,3 & 29,1 \\
\hline Autoeficacia & 58,1 & 29,4 \\
\hline Aprendizaje & 54,7 & 26,9 \\
\hline Generatividad & 60,4 & 27,6 \\
\hline Puntaje Total & 56.3 & 25.1 \\
\hline
\end{tabular}

\subsection{ESTUDIO DE CORRELACIONES ENTRE LAS VARIABLES}

En el cuadro 5 se puede observar las distintas relaciones entre las variables. Aparece una correlación positiva significativa entre las variables Rendimiento escolar y Rendimiento 
intelectual (.21*). Además, se evidencia una correlación entre los niveles de Autoestima de los estudiantes y el Bienestar psicológico (.40**). Entre las variables Autoestima y Resiliencia, aparece también una correlación significativa importante $(.51 * *)$. Finalmente los resultados indican que el Bienestar Psicológico se relaciona con la variable resiliencia $\left(.47^{* *}\right)$.

Las relaciones entre las dimensiones de la escala de bienestar psicológico y los subfactores de la escala de Resiliencia, se evidencia en: Control e Identidad (.33**), Control y Auto eficacia (.31**), Control y Generatividad (.33**). Con respecto a la dimensión Vínculos, se aprecia que ésta se correlaciona con Afectividad (.26**). La dimensión Proyecto se correlaciona con Autonomía (.22*). Finalmente, la dimensión autocontrol se correlaciona con Pragmatismo (.26**).

La relación existente entre las dimensiones del Bienestar psicológico se da solo con el factor General de la escala de Autoestima: Control (034**), Vínculo (.19*), Proyectos (.23*) y Aceptación (.25**).

Respecto a la relación de los factores de la escala de autoestima y las dimensiones de Resiliencia, se encontró que existen correlaciones significativas entre el factor General de la escala de Autoestima e identidad, (.33**), Autonomía (.35**), Vínculos (.31**), Metas $\left(.33^{* *}\right)$, Afectividad $(.34 * *)$ y Auto eficacia $\left(.35^{* *}\right)$. Todas ellas son dimensiones de la escala de Resiliencia.

El factor Social de la escala de Auto estima se correlaciona con Vínculos (.25**). El factor Hogar se correlaciona con Vínculo (.31**), Redes $(.37 * *)$ y Modelos $\left(.33^{* *}\right)$ $\mathrm{y}$, finalmente, el factor Escolar se correlaciona con la dimensión Social y Metas $\left(.26^{* *}\right)$ y $(.28 * *)$ respectivamente.

Cuadro 5. Correlaciones entre variables

\begin{tabular}{|c|c|c|c|c|c|}
\hline & $\begin{array}{c}\text { Rendimiento } \\
\text { Escolar }\end{array}$ & Autoestima & $\begin{array}{c}\text { Rendimiento } \\
\text { Intelectual }\end{array}$ & Bieps & Resiliencia \\
\hline $\begin{array}{c}\text { Rendimiento } \\
\text { Escolar }\end{array}$ & 1 & .028 & $.21^{*}$ & -.035 & -0.15 \\
\hline Autoestima & & 1 & -.024 & $.40^{* *}$ & $.51^{* *}$ \\
\hline $\begin{array}{c}\text { Rendimiento } \\
\text { Intelectual }\end{array}$ & & & 1 & -.061 & -.137 \\
\hline Bieps & & & & 1 & $.47^{* *}$ \\
\hline Resiliencia & & & & & 1 \\
\hline
\end{tabular}

\subsection{ANÁLISIS COMPARATIVO ENTRE LOS ALUMNOS CON DISTINTOS RENDIMIENTO ESCOLAR}

La comparación entre los distintos grupos se puede apreciar en el Cuadro 5. Para las distribuciones normales se aplicó análisis de las varianzas, mientras que aquellas distribuciones no paramétricas la prueba estadística aplicada fue Kruskal-Wallis (W). Con respecto al rendimiento intelectual, las diferencias son significativas: se obtiene un F 5,8 $(\mathrm{p}<01)$. Cabe destacar que todos los puntajes del rendimiento intelectual se encuentran dentro de la norma estadística, sin embargo, las diferencias se encuentran entre el grupo 
de rendimiento alto (RA) con el rendimiento promedio (RP) y el bajo (RB), no hay diferencias entre el grupo promedio (RP) con el bajo (RB). Respecto a las diferencias en la variable autoestima, bienestar psicológico y resiliencia no se evidencia diferencias significativas entre los grupos.

Cuadro 6. Análisis de los promedios de cada una de las variables

\begin{tabular}{|c|c|c|c|c|c|}
\hline & $\begin{array}{c}\text { Rendimiento } \\
\text { Intelectual }\end{array}$ & Autoestima & Bieps & Resiliencia & $\mathrm{N}$ \\
\hline R.A*. Media & 45,5 & 52,81 & 34,69 & 49,33 & 33 \\
$(>=6,0)$ D.E & 3,78 & 9,37 & 2,55 & 24,2 & \\
\hline R. P. Media & 41,66 & 51,01 & 34,78 & 53,65 & 120 \\
(5,9-5,0) D.E. & 6,10 & 9,87 & 4,07 & 25,7 & \\
\hline R. B. Media & 39,8 & 49,36 & 35,00 & 59,66 & 22 \\
(<=4,9) D.E. & 7,19 & 10,7 & 2,60 & 22,2 & \\
\hline F & $5,8^{* *}$ & 2.31 (n.s.) & 0.56(n.s.) & 2.42 (n.s.) & 175 \\
W & & & & & \\
\hline
\end{tabular}

Rendimiento Alto (R.A.), Rendimiento Promedio, (R.P), Rendimiento Bajo (R.B.)

Respecto a un análisis más fino de las distintas sub escalas de la variable autoestima (cuadro 7), no se evidencia diferencias significativas en la percepción que tienen los diversos grupos de sí mismo y de su autovaloración personal. La imagen que perciben que sus padres poseen de ellos, la autovaloración y valoración respecto a su rendimiento escolar y la autoevaluación que hacen respecto a su desempeño social como el grado de aceptación que perciben del grupo en general, no son características que nos permiten distinguir a los grupos.

Cuadro 7. Resultado del Inventario de Autoestima y sus diferentes escalas

\begin{tabular}{|c|c|c|c|c|}
\hline & General & Social & Hogar & Escolar \\
\hline R.A*. Media & 52,0 & 51,51 & 48,33 & 55,57 \\
(>=6,0) D.E & 8,27 & 14,63 & 10,40 & 9,46 \\
\hline R. P. Media & 50,46 & 55,2 & 45,87 & 55,15 \\
(5,9-5,0) D.E. & 9,20 & 11,02 & 7,94 & 10,42 \\
\hline R. B. Media & 48,86 & 54,13 & 46,00 & 49,86 \\
(<=4,9) D.E. & 8,89 & 10,9 & 8,62 & 12,04 \\
\hline$W$ & 0.965 & 0.92 (n.s.) & 1.96 (n.s.) & 4.93 (n.s.) \\
& (n.s.) & & & \\
\hline
\end{tabular}

Con relación al Bienestar Psicológico (cuadro 8), los resultados indican que no existen diferencias significativas entre los grupos en las dimensiones: Control, vínculos y en proyecto. Sin embargo, existen diferencias significativas en lo que se refiere a la autonomía, la cual se relaciona con la toma de decisiones de modo independiente, confiar en su propio juicio, estabilidad emocional, expresar lo que se piensa sin problemas y hacer lo que se quiere sin ser influenciable. Estas habilidades se expresan con un puntaje 
mayor en el grupo que presenta un Rendimiento Bajo (RB.) y Rendimiento Promedio (R.P.) que en el grupo de Rendimiento Alto (RA).

Cuadro 8. Bienestar Psicológico y sus diferentes Dimensiones

\begin{tabular}{|l|l|l|l|l|}
\hline & Control & Vínculo & Proyecto & Autonomía \\
\hline R.A.*. Media & 10,72 & 8,00 & 8,54 & 7,51 \\
(>=6,0) D.E & 1,25 & 1,06 & 0,86 & 1,34 \\
\hline R. P. Media & 10,67 & 8,04 & 8,26 & 8,12 \\
(5,9-5,0) D.E. & 1,20 & 1,01 & 1,01 & 1,08 \\
\hline R. B. Media & 10,68 & 8,13 & 7,95 & 8,22 \\
(<=4,9) D.E. & 1,08 & 0,83 & 1,32 & 0,81 \\
\hline \multicolumn{1}{|c|}{$W$} & 0.19 (n.s.) & 0.064 (n.s.) & 3.82 (n.s.) & 7.02 (sig.) \\
\hline
\end{tabular}

Finalmente, respecto a las diferentes dimensiones de la escala de Resiliencia, la diferencia se evidencia en el sub escala de satisfacción; esto se relaciona con la percepción de logro, autovaloración, adaptación efectiva a las condiciones ambientales y percepción de desarrollo. Los estudiantes de rendimiento promedio alcanzan un puntaje más alto respecto a los grupos con rendimiento alto y bajo. Una observación interesante es que el grupo de rendimiento bajo (RB), las puntuaciones en casi todas las dimensiones alcanzan mayores puntuaciones que los demás grupos.

Cuadro 9. Escala de Resiliencia para jóvenes y adultos, (SV-RES)

\begin{tabular}{|l|l|l|l|l|l|l|}
\hline & Identidad & Autonomía & Satisfacción & Pragmatismo & Vínculos & Redes \\
\hline R.A*. Media & 56.77 & 60,30 & 41,14 & 55,70 & 53,38 & 49,26 \\
(>=6,0) D.E & 26,22 & 25,33 & 26,17 & 25,26 & 29,57 & 29,48 \\
\hline R. P. Media & 60,75 & 61,42 & 54,51 & 55,76 & 53,50 & 55,85 \\
(5,9-5,0) D.E. & 26,75 & 27,86 & 26,28 & 27,58 & 27,97 & 28,48 \\
\hline R. B. Media & 62,1 & 59,29 & 49,49 & 65,54 & 56,61 & 63,90 \\
(<=4,9) D.E. & 21,56 & 25,18 & 24,34 & 23,78 & 26,40 & 21,82 \\
\hline \multicolumn{1}{|c|}{$W$} & 0.81 (n.s.) & 0.21 (n.s.) & $6.49(\mathrm{~s})$ & 2.17 (n.s.) & 0.20 (n.s.) & 3.33 (n.s.) \\
\hline \multicolumn{6}{|l|l|l|l|l|l|l|l|}{} \\
\hline
\end{tabular}

\section{CONCLUSIÓN Y DISCUSIÓN}

El objetivo de este estudio fue analizar el alto rendimiento que presentaba un grupo de estudiantes de cuarto año medio de liceos con altos índices de vulnerabilidad en comparación con sus grupos cursos. Se estudiaron diversas variables relacionadas con rendimiento intelectual, autoestima, bienestar psicológico y resiliencia. Se estudiaron en una primera etapa las relaciones entre variables, donde se evidenció que entre el rendimiento escolar y el rendimiento intelectual existe una correlación débil positiva significativa, lo que se confirma en otros estudios (Sternberg y Castejón, 1999 y Canales, 
2008). Con respecto a las otras variables no se encontraron correlaciones significativas con el rendimiento escolar. Sin embargo, entre la autoestima, el bienestar psicológico y la resiliencia los resultados arrojan correlaciones significativas. Entre los subfactores de las distintas escalas, se establece una relación entre las sub escalas control y la identidad; autoeficacia y la generatividad. Aquellos estudiantes que poseen auto competencia, manejar los asuntos de la vida diaria, conciencia de las oportunidades y capaces de modificar el ambiente son también jóvenes que se definen relativamente estables en la interpretación de los hechos, capaces de reconocer en sí mismos posibilidades de éxitos en situaciones problemáticas y solicitar ayuda frente a situaciones complejas.

Aquellos estudiantes con puntuaciones altas en el sub test de vínculos, expresada en capacidad de aceptar aquellos aspectos positivos como negativos de sí mismos también manifiestan actitudes positivas de afectividad, relacionadas con el entorno.

La relación entre la dimensión proyecto y autonomía también se presenta como una relación positiva; aquellos jóvenes que poseen proyectos y metas definidas poseen también determinados juicios para definir su aporte a su entorno sociocultural. La autonomía también se relaciona con el pragmatismo: son jóvenes que generan juicios que develan la forma de interpretar las acciones que realizan. Las investigaciones coinciden con estos resultados: la autoestima es uno de los predictores de mayor fuerza y consistencia de bienestar psicológico (Diener, 1994). Los estudios de Dew y Scott (2002) muestran que los adolescentes que lograban un alto grado de satisfacción, expresan un alto grado de autoestima y poseen un mayor control de los sucesos vitales.

Con relación a las comparaciones entre los grupos de alto y bajo rendimiento, los resultados indican que las diferencias se encontraron en el rendimiento intelectual. Aunque ésta capacidad en los estudiantes de alto rendimiento no se encuentra en un puntaje alto, sí podríamos señalar que poseen una media superior al grupo. Respecto a la variable autoestima, se evidencia que no existe diferencia entre los grupos. Las subescalas del inventario de autoestima muestran que el comportamiento de los grupos en la percepción de sí mismo, respecto a la familia, a la escuela y frente a sus amigos, no se diferenciaron.

Por su parte, respecto al bienestar psicológico, las diferencias sólo se expresan en la dimensión de autonomía, relacionada con la capacidad para la toma de decisiones de modo independiente, la confianza que poseen los sujetos de su propio juicio, la estabilidad emocional, la capacidad para expresar lo que se piensa sin problemas y hacer lo que se quiere sin ser influenciable. Estos aspectos aparecen en el grupo de bajo rendimiento quienes lograron alcanzar una puntuación mayor que aquellos que poseen un rendimiento escolar más alto.

La variable resiliencia -entendida como la capacidad que poseen los estudiantes para proyectarse en el futuro, a pesar de sucesos o situaciones agravantes, desestabilizadoras, de condiciones complejas, vulnerables y a veces de situaciones traumáticas graves- no logra diferenciar los diversos grupos. La significatividad sólo se puede observar en la sub escala satisfacción, aspecto que tiene que ver con la percepción de logro, autovaloración, adaptación efectiva a las condiciones ambientales y percepción de desarrollo. El grupo promedio obtiene puntajes más altos que los demás grupos. Por su parte, el puntaje menor es el grupo de rendimiento alto.

Los resultados obtenidos de esta investigación logran entregar información que contradice otros estudios. En general, los reportes muestran una relación positiva y significativa entre autoestima y rendimiento escolar y era también esperable que aquellos estudiantes 
que poseían alto rendimiento escolar presentaran un bienestar psicológico mayor que aquellos estudiantes con un bajo rendimiento.

Estos datos deben interpretarse dentro de un contexto político, social y económico que nuestro país está viviendo, específicamente frente al movimiento que hoy día están viviendo lo estudiantes con respecto al sistema educativo. Los estudiantes que participaron en la investigación son jóvenes de 17-19 años, del último año de la enseñanza media, con buen rendimiento escolar, perteneciente al I y II quintil (niveles per cápita familiar), donde su acceso a la educación superior en Chile no es fácil. Aunque la cobertura de educación superior, durante los últimos años, ha aumentado desde un $27,4 \%$ a un $29,1 \%$ entre el 2006 y el 2009, para los jóvenes entre 18 y 24 años de edad, para aquellos jóvenes que se sitúan en el $10 \%$ de menores ingresos la tasa de acceso, ha aumentado desde un $12,3 \%$ a un $16,4 \%$ entre el 2006 y el 2009 , mientras que para los jóvenes del $10 \%$ de mayores ingresos el aumento ha sido desde 59,5\% a 61,5\% para el mismo período (Ministerio de Planificación, 2011).

Esta realidad que viven los jóvenes con alto rendimiento escolar pertenecientes a sectores vulnerables, los deja más expuestos a estados de frustración, ansiedad e inseguridad, debido que a pesar que ellos desean insertarse a la universidad o iniciar estudios de educación superior, su acceso les es difícil producto de múltiples factores que contribuyen a que su proyecto de vida se transforme en algo inestable, impredecible e inseguro, interfiriendo en su bienestar psicológico.

Aquellos estudiantes que poseen un rendimiento promedio o bajo, probablemente no tendría mayores pretensiones de llegar a la Universidad, por lo cual su bienestar aparecería con mejores puntuaciones.

Con respecto a la resiliencia, algunos estudios han arrojado correlaciones negativas con el rendimiento escolar. Para que se manifiesten en los estudiantes los comportamientos resilientes deben existir situaciones o sucesos que hayan afectado al sujeto y éstas hayan sido enfrentadas adecuadamente. Aquellos estudiantes que presentan alto rendimiento, la tendencia es que expresen baja puntuación en la escala de resiliencia, mientras que los que presentan bajos rendimientos tendrían mayores conductas resilientes. Por su parte, la resiliencia es una variable resultante de varios factores, por lo cual sería necesario analizar cada uno de ellos de manera más focalizada.

Hay muchos aspectos que son necesarios analizar. Las motivaciones que presentan los estudiantes tendrían una relación fundamental en los logros académicos. Las bajas expectativas que los docentes tienen de los estudiantes, según Bisquerra (2006), generan bajo rendimiento en los estudiantes y fracaso escolar y, por otra parte, dificultad para controlar las clases y los problemas de disciplina. La hipótesis que pudiese confrontarse es que aquellos estudiantes que poseen alto rendimiento, presentarían una motivación para aprender, disposición al esfuerzo deseo de conseguir una calificación positiva y deseo de éxito y reconocimiento.

Durante los últimos años se han desarrollado varias investigaciones sobre el bienestar psicológico en jóvenes insertados en el sistema escolar. Existen personas que se sienten muy bien y otros no tan bien, la pregunta central es: ¿qué genera esta diferencia? O: ¿qué factores incidirían y cómo esto se relacionaría con otras variables? Y, finalmente, ¿cuál es su repercusión con el rendimiento escolar? Autores que han tratado de buscar respuesta, tales como Casullo y Castro Solano (2000), Diener, Suh y Oishi (1997), González, Barrón, Montoya Castilla Casullo y Verdú (2002) y Ryfff (1989), se preguntan si son factores de 
personalidad o factores de contexto los que tendrían una mayor incidencia en como los sujetos construyen una auto percepción de sí mismo y de su bienestar subjetivo.

Los estudios realizados por Veenhoven (1994) sostienen que el bienestar psicológico sería superior en los países que tienen mejores condiciones de vida, por lo cual esto pudiese también transferirse a contexto socioculturales. Aquellos estudiantes que se insertan en instituciones escolares con mayores oportunidades y condiciones, alcanzarían un mayor bienestar. Sin embargo, en aquellos sujetos que han logrado satisfacer las necesidades básicas, no se evidenciarían diferencias en su bienestar subjetivo entre los grupos sociales.

Por otra parte, el bienestar psicológico podría relacionarse con los sucesos vitales normativos y no normativos que los adolescentes han tenido que enfrentar y cómo éstos influyen en diversas dimensiones del bienestar, entre las cuales, como mencionan Aspinwall y Staudinger (2007), se encuentra el dominio del medio ambiente, relaciones positivas, propósitos en la vida, auto aceptación, crecimiento personal y autonomía, dimensiones que se deben trabajar durante todo el proceso escolar, con el propósito de instaurar competencias que el joven adolescente pueda incorporar a su vida para enfrentar las situaciones diversas y adversas que debe confrontar durante las distintas etapas del desarrollo.

La realidad que viven los estudiantes de alto rendimiento que estudian en instituciones escolares vulnerables, en general, se caracteriza por un comportamiento estresado. Figueroa y Cohen (2006) señalan que el estrés sería el resultado de una relación particular entre la persona y el contexto, un proceso evaluativo que el sujeto realiza del estímulo como amenazante o desbordante para sus recursos. En la etapa de la adolescencia, producto de una serie de cambios, pueden generar desequilibrio entre el individuo y el entorno. En consecuencia, es fundamental conocer cómo estos sujetos logran afrontar estas situaciones adversas y si existirían diferencias entre grupos de alto rendimiento con aquellos de bajo rendimiento escolar. La hipótesis que se sostendría es que la presencia de apoyo social y familiar en los estudiantes de buen rendimiento escolar, lograrían estar en mejores condiciones para usar estrategias de afrontamiento más adaptativo a los estímulos estresores, por lo cual lograrían mantener su rendimiento; mientras que aquellos que no se evidencia esta figura de un profesor, de un familiar, de un amigo cercano estaría con mayor riesgo de no presentar una mayor estabilidad psicológica, lo que podría provocar situaciones de inestabilidad en el plano emocional.

Finalmente, el uso de una metodología cualitativa, utilizando técnicas de entrevistas en profundidad con los estudiantes de alto rendimiento escolar, pudiese ser una adecuada aproximación para comprender y reconstruir que factores han sido los responsables de los altos rendimientos escolares.

\section{REFERENCIAS BIBLIOGRÁFICAS}

Amar, J., Kotliarenko, M. A. y Abello, R. (2003). Factores psicosociales asociados con la resiliencia en niños colombianos, víctimas de violencia intrafamiliar. Investigación y Desarrollo, vol.11, n. 1, $162-197$

Aspinwall, L. y Staundinger, U. (2007). Psicología del Potencial Humano. Barcelona: Gedisa

Bisquerra, R.,(2006). Educación emocional y bienestar. Madrid: Praxis

Canales, R. (2008). Procesos Cognitivos y estrategias psicolingüísticas que intervienen en la lectura comprensiva, diseño y ejecución de un programa experimental en niños con problemas de aprendizaje. Revista de Investigación en psicología, vol.11, n.1, 81-100 
Castro, A. (ed.) (2010). Fundamentos de Psicología Positiva. Buenos Aires: Paidós

Casullo, M.M. y Castro Solano, A. (2000). Evaluación del Bienestar Psicológico en estudiantes adolescentes argentinos. Revista de Psicología, vol.XVIII, n.2, 25-49

Casullo, M. M. (2000). Psicología salugénica o positiva. Algunas reflexiones. VIII Anuario de Investigaciones. Buenos Aires: Facultad de Psicología. UBA.

Csikszentmihalyi, M. (1999). If we are so rich why aren`t we happy? American Psychologist, vol.54, n.10, 821-827.

Dew, T. y Scott, E. (2002). La calidad de vida percibida en los adolescentes: una investigación exploratoria. Columbia: Universidad de Carolina del Sur.

Diener, E. (1994). El bienestar subjetivo. Barcelona: Paidós.

Diener, E.; Suh, E. \& Oishi, S. (1997). Recent Finding on Subjective Well-Being. Indian Journal of Clinical Psychology. Workshop on "Measuring Empowerment: Cross-Disciplinary Perspectives" held at the World Bank in Washington, DC on February 4 and 5, 2003.

Figueroa, M.I. y Cohen, S. (2006). Estrategias y estilos de afrontamiento del estrés en adolescentes. En Contini, E. (Ed.), Pensar la adolescencia hoy. De la psicopatología al bienestar psicológico. Buenos Aires: Paidós

García, B..(1983). Análisis y delimitación del constructo autoestima. Madrid: Editorial de la UCM.

González, Barron, R.; Montoya Castilla, I.; Casullo, M.M.; Verdú, J.B. (2002). Relación entre estilos y estrategias de afrontamiento y bienestar psicológico en adolescentes. Psicotema, vol.14, n.2, 363-368

Grotberg, E. (1995). A guide to promoting resilience in children:Streng - hening the human spirit. The Instrumental Resilience Proyect. Bernard Van Leer Foundation. Holanda: La Haya.

Henderson, N. y Milstein, M. (2003). Resiliencia en las escuelas. Buenos Aires: Editorial Paidós.

Luthans, F. (2002). The need for and meaning of positive organizational behavior. Journal of Organizational Behavior, vol.23, 695-706.

Masten A.S.(2001) Ordinary magic. Resilience processes in development. American Psychologist, n.56, 227-239.

Mruk, C. (1999). Self-esteem research, theory and practice. New York: Springer Publishing Company.

MINEDUC (2006a). Especificaciones técnicas y administrativas para la asistencia técnica de las universidades a los liceos prioritarios. Santiago: Mineduc.

Ministerio de Planificación. (2011). Encuesta de caracterización socioeconómica 2009. Santiago, Chile: Ministerio de Planificación.

Naranjo, M. (2007). Autoestima: Un factor relevante en la vida de la persona y tema esencial del proceso educativo. Revista Electrónica Actualidades Investigativas en Educación, vol. 7, n.3.

Padierna, L.; Oseguera, J.; y Gudino, N. (2009). Factores socioacadémicos, estilo de aprendizaje, nivel intelectual y su relación con el rendimiento académico previo de médicos internos de pregrado. Educación Médica. [Online], vol.12, n.2, 91-102. ISSN 1575-1813. http://dx.doi. org/10.4321/S1575-18132009000300005.

Polleti, R. y Dobbs, B. (2005). La autoestima. Un bien esencial. Buenos Aires: Lumen.

Ramos, M. (2004). Valores y autoestima. Conociéndose a sí mismo en un mundo de otros. Revista Educación en Valores, vol.1, n.1.

Ryff, C. (1989). Happiness is everything, or is it? Exploratioms on the meaning of psychological well-being. Journal of Personality and Social Psychology, vol.57, n.6, 1069-1081

Romero, O. (2005). Competencias para el crecimiento psicológico. Icone Educacao, vol.11, n.2, 1-5

Rutter, M. (1985). Resilience in the face of adversity protective factors and resistance to psychiatric disorder. British Journal of Psychiatry, vol.1, n. 47, 598 - 611

Saavedra, E. y Villalta, M. (2008). Escala de Resiliencia SV-RES. Para jóvenes y adultos (2 ${ }^{a}$ edición). Santiago de Chile: CEANIM.

Sternberg, R. y Castejón J. (1999).Estilo Intelectual y Rendimiento académico. Revista de Investigación Educativa, vol. 17, n.1, 33-46

Veenhoven, R. (1994). El estudio de la satisfacción con la vida. Intervención Psicosocial, vol.3, n.9, 87-116. 